\title{
Do Palm Cockatoos (Probosciger aterrimus) have long enough lifespans to support their low reproductive success?
}

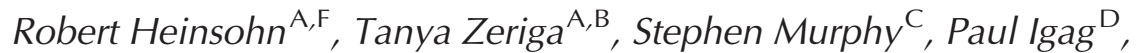 \\ Sarah Legge ${ }^{\mathrm{C}}$ and Andrew L. Mack ${ }^{\mathrm{E}}$

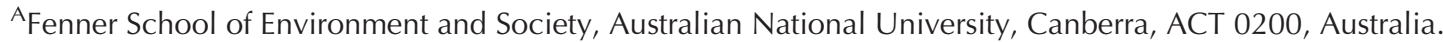 \\ ${ }^{B}$ Wildlife Conservation Society, PO Box 277, Goroka, EHP, Papua New Guinea. \\ ${ }^{\mathrm{C} A u s t r a l i a n}$ Wildlife Conservancy, Suite 5 Hay Street, Subiaco, WA 6008, Australia. \\ Dapua New Guinea Institute of Biological Research, Goroka, EHP, Papua New Guinea. \\ EPowdermill Nature Reserve, 1847 Rte 381, Rector, PA 18677, USA. \\ FCorresponding author. Email: Robert.Heinsohn@anu.edu.au
}

\begin{abstract}
As an order, Parrots (Psittaciformes) are both the longest lived birds and among the most endangered. Palm Cockatoos (Probosciger aterrimus) have an extremely slow life history, including one of the lowest rates of breeding success reported for parrots. Here we use population viability analysis (PVA) to examine whether populations of Palm Cockatoos are viable in the long term with such low rates of reproduction. We built PVA models for two field sites with robust data on reproductive success and availability of nest-hollows: Iron Range on Cape York Peninsula (Queensland, Australia) and Crater Mountain (Eastern Highlands Province, Papua New Guinea). Our Iron Range models incorporate a fluctuating resource base of nest-hollows resulting from natural losses from fire, wind and tree decay and natural replenishment through tree breakages in tropical cyclones. The number of females allowed to breed each year in the simulations was limited by the availability of hollows. Our Iron Range models suggest that the population is only viable if juvenile and adult mortality rates are very low, and that at least some birds in the population live to 100 years old. Based on known maximum lifespans in the Cacatuidae, we conclude that Palm Cockatoos at Iron Range are unlikely to live for long enough on average to support their low rates of reproduction, and highlight the possibility that the population may be in decline. At Crater Mountain, where nestlings are harvested for food by local people, the models suggest that the reported current rate of $40 \%$ of nestlings taken is not sustainable.
\end{abstract}

\section{Introduction}

Birds are generally long-lived and slowly ageing animals. They may live up to three times longer than mammals of equivalent body mass, despite much higher metabolic rates and total lifetime energy expenditure (Austad 1993). However, maximum lifespan varies considerably among birds. Galliformes, including poultry, are the shortest lived, whereas parrots (Psittaciformes), seabirds (Charadriiformes), songbirds (Passeriformes), hummingbirds (Apodiformes) and raptors (Falconiformes) all include species with exceptionally long lifespans for their body size (Holmes et al. 2001). The oldest known wild birds come from banding studies of seabirds, for example a Manx Shearwater (Puffinus puffinus) in Britain and a Southern Royal Albatross (Diomedea epomophora) in New Zealand both exceeded 50 years of age. Evolutionary theories of ageing suggest that the intrinsic lifespan of a species should increase as the rate of extrinsic mortality decreases (Austad and Fischer 1991). Higher rates of mortality from extrinsic factors such as predation or disease are believed to decrease intrinsic lifespan because late-acting deleterious alleles are not as exposed to natural selection. The ability of birds to fly, and therefore escape predators more easily, may contribute to their low rates of extrinsic mortality.
As an order, the Psittaciformes are the longest-lived birds for their body size (Prinzinger 1993). Cockatoos (Cacatuidae) have higher maximum longevity than parrots (Psittacidae and Loriidae; mean maximum ages 39.5, 22.7 and 17.0 years respectively). The two genera with the longest-lived species are cockatoos Calyptorhynchus and Cacatua (mean maxima of 46.0 and 39.5 years respectively) (Munshi-South and Wilkinson 2006). However, some of the older ages reported in the literature, for example of parrots living for more than 80 years, are difficult to substantiate. A review of captive records until 1997 showed that the oldest official record was for a Salmon-crested Cockatoo (Cacatua moluccensis) that died aged at least 69 years, whereas a Major Mitchell's Cockatoo (Lophochroa leadbeateri) was still alive aged 63 years (Brouwer et al. 2000).

The Psittaciformes are also one of the most endangered orders of birds, with at least $29 \%$ of species worldwide considered to be threatened (Snyder et al. 2004; BirdLife International 2009). The greatest threats are usually from habitat loss, hunting and capture for the pet trade, but these may be compounded by slow life histories, including low reproductive rates over long lifespans. Over half of all species of cockatoo are believed to be endangered (Juniper and Parr 1998; Snyder et al. 2004). 
Most studies of tropical species of cockatoos have consisted of surveys to assess population size and breeding activity (Walker et al. 2005; Cahill et al. 2006). One species that has been studied in greater detail is the Palm Cockatoo (Probosciger aterrimus) a sedentary species that occurs in lowland New Guinea and its offshore islands, the Aru Islands, and far northern Cape York Peninsula in Australia. Globally, Palm Cockatoos are under threat from hunting, the pet trade, land clearing and changes to fire management. This charismatic species has been the subject of two major field studies, one at Iron Range on Cape York Peninsula, Australia, from 1999 to 2002 (Murphy et al. 2003) and another at Crater Mountain, in the Eastern Highlands Province of Papua New Guinea (PNG) from 1999 to 2001 (Igag 2002). Both studies documented habitat preferences and breeding biology, including nest-hollow preferences, site-fidelity, and long-term breeding success. Igag (2002) also assessed the rates at which local people harvested nestlings for food. Palm Cockatoos were found to have an extremely slow life-history. Females lay only one egg per clutch, and those at Iron Range were recorded only to do so every 2.2 years on average. A low probability of fledging success, mainly owing to infertility and predation, means that nesting pairs only fledge a mean of 0.11 offspring per year (Murphy et al. 2003).

Palm Cockatoos clearly must live to great ages if they are to sustain their population levels with such low rates of reproduction. However, longevity data, especially from longlived species, are difficult to acquire from field studies. Here we apply an alternative approach, using population viability analysis (PVA). PVAs are computer simulation models that use demographic data to make quantitative predictions about population size over time and the likelihood of extinction (Beissinger and Westphal 1998; Miller and Lacy 2005). We use this approach to assess three key questions relating to the viability of Palm Cockatoo populations in the wild. First, what must the maximum lifespans and population age-structures be for Palm Cockatoo populations to remain viable? Second, are such lifespans likely, given our understanding of the ages birds reach in the wild? Third, given the extended lifespan and low reproductive rate of Palm Cockatoos, is it possible to sustainably harvest the nestlings for food as occurs in many areas of New Guinea? We use PVA as an exploratory tool to gather insights into the demographic structure and conservation status of a bird that may prove either to be one of the longest lived species or a species in serious decline, or both.

\section{Methods}

\section{Field sites and data sources}

We used data from two field studies of Palm Cockatoos, a fouryear study at Crater Mountain, PNG (Igag 2002) and a threeyear study at Iron Range National Park on Cape York Peninsula, Australia (Murphy et al. 2003), to run computer simulations of their likely age-structure and long-term population viability. Iron Range National Park is situated on Cape York Peninsula in far northern Queensland $\left(12^{\circ} 47^{\prime} \mathrm{S}, 143^{\circ} 18^{\prime} \mathrm{E}\right)$ and contains the largest remaining area of lowland rainforest in Australia. Much of the rainforest is distributed patchily in a mosaic with other vegetation types such as eucalypt woodland and heath. Palm
Cockatoos occur only in limited areas of northern Cape York Peninsula and prefer to nest in woodland trees within $300 \mathrm{~m}$ of the rainforest boundary. Nesting in woodland allows Palm Cockatoos to have access to a greater number of hollows while remaining close to their rainforest food sources. The major competitors for nest-hollows are Sulphur-crested Cockatoos (Cacatua galerita) (Heinsohn et al. 2003), and the major nest-predators include varanid lizards (Varanus spp.), Giant White-tailed Rats (Uromys caudimaculatus), Black Butcherbirds (Cracticus quoyi) and Amethyst Pythons (Morelia amethistina) (Murphy et al. 2003). The Iron Range population is probably contiguous with that of the McIlwraith Range to the south. The combined population may be isolated from other populations on Cape York Peninsula although there may be some connection between populations via the gallery forests of major rivers such as the Wenlock and Archer Rivers.

In New Guinea, Palm Cockatoos are found in hill and lowland rainforests. In southern New Guinea they may occur in mosaics of woodland and rainforest as on Cape York Peninsula, but they also occupy large intact tracts of rainforest, possibly at lower densities. The data used in this study are from the Crater Mountain Wildlife Management Area (CMWMA), located in continuous forest that is inhabited by people on the southern slopes of the central ranges $\left(6^{\circ} 43^{\prime} \mathrm{S}, 145^{\circ} 05^{\prime} \mathrm{E}\right)$ between 100 and $1200 \mathrm{~m}$ above sea level (Igag 2002). The Pawaia, Gimi and other people at Crater Mountain maintain food gardens in this region and also harvest Palm Cockatoo nestlings among many other animals (Mack and West 2005).

\section{Modelling approach and parameters}

We used VORTEX software (Miller and Lacy 2005) to model population trajectories via the discrete, sequential events that happen to individuals (e.g. births, deaths, reproduction, environmental changes) according to defined probabilities determined from field data. VORTEX is an individual-based simulation of the deterministic and stochastic forces affecting populations, and is primarily used to model the probability of extinction of small populations and the relative effects of differing treatments and perturbations. The probabilities of events are modelled as constants or as random variables that follow specified distributions. Since the growth or decline of a simulated population is strongly influenced by these random events, separate model iterations using the same input produce different results. Consequently the model is repeated many times (e.g. 1000 repetitions) to reveal the distribution of fates that the population might experience under a given set of input conditions. VORTEX also models catastrophes as random events that affect survival and reproduction, and simulates the transmission of genes to determine if inbreeding depression decreases population viability. Outputs include summary statistics on population growth rates, the probability of population extinction over a specified period, the time to extinction, and the mean size and genetic variation in extant populations.

No complete population censuses have been attempted for either the Iron Range or Crater Mountain populations. We stress that we are more concerned with the population dynamics owing to low reproductive success and limited availability of nest- 
hollows than with starting population size. We used a starting population of 1000 Palm Cockatoos (500 males, 500 females), with a carrying capacity of 2000 , in all simulations in both regions. We estimated this number by extrapolating the number of displaying males $\left(4.4 \mathrm{~km}^{-2}\right)$ and nest-hollows (3.4 per male) observed by Murphy et al. (2003) in one area of the Iron Range National Park to the entire area of likely breeding habitat in the Iron Range region ( $\sim 11000$ ha of rainforest-woodland ecotone; Nelder and Clarkson 1995). We also examined the sensitivity of this variable by running the models with a starting population of 500 Palm Cockatoos. Although the Crater Mountain population is likely to be continuous with surrounding areas, we used the same starting population size to allow comparisons with Iron Range. Simulations were run 1000 times, the time frame used was 100 years, and extinction was taken as occurring when only one sex remained. VORTEX models require an estimate of the level of concordance between reproductive success and mortality. We assumed low concordance based on field observations that Palm Cockatoos often failed to breed but with no apparent effect on their survival.

\section{Reproductive success, sexual maturity and mating system}

We used the data on reproductive rates and success outlined in Murphy et al. (2003) and Igag (2002). Data from Iron Range were attained by the close monitoring of 28 active nests, including 41 actual breeding attempts, over three years. The females in this study had at most one young per year but were only observed to attempt to breed every 2.2 years on average. They lay just one egg, which has a low probability $(0.22)$ of hatching and fledging. A small number of females lay again in the same season if their first attempt fails, leading to an overall probability of fledging one young over a single season of 0.25 for a single season. We combined the probability of attempting to breed with the probability of success into one annual figure of 0.11 . Breeding success is higher for Crater Mountain, with a probability of fledging one chick of 0.40 , but their overall reproductive rate is less clear (see below; Igag 2002).

The ages of sexual maturity and maximum age of reproduction for wild Palm Cockatoos are not known. Palm Cockatoos in captivity have not been observed breeding before the age of seven or eight years old. One captive female was reported to be at least 40 years old before she laid her first egg, suggesting that the captive environment is not conducive to early breeding (Brouwer et al. 2000). Black-cockatoos (Calyptorhynchus sp.) appear to reach sexual maturity earlier than this in the wild. For example, the similarly sized Carnaby's Black-Cockatoo (Capyptorhynchus latirostris) from Western Australia first attempt to breed at four years old (Saunders 1982, 1986). Consequently, we use four years as the age of sexual maturity for both sexes in our models, but test the sensitivity of this variable by examining the effect on population viability if the age of first breeding in wild Palm Cockatoos is in fact higher. In the models, we have also allowed a generous maximum longevity of 150 years, with no reproductive senescence. This does not assume that they live that long but it does allow computation of the mortality schedules that would be necessary for the populations to remain viable given the low rates of reproduction outlined above (see 'Mortality', below).

We ran our models using 'long-term monogamy' as the mating system, as DNA fingerprinting showed that breeding at Iron Range was mostly monogamous over multiple years (Murphy et al. 2003). Under the long-term monogamy option in VORTEX, the same individuals continue to breed exclusively with each other until one of them dies. Although available sample sizes are small there is no evidence that the hatching sex-ratio differs from parity. All demographic values used in our PVA models are shown in Table 1.

\section{Mortality}

There are no data on age-specific mortality for wild Palm Cockatoos. We took the alternative approach of initially assuming that the population is stable (neither increasing nor decreasing), and that reproduction and mortality must therefore balance each other. We used the known low reproductive rate at Iron Range to infer the mortality schedules and longevity that would be necessary for this to be true. This allows us to evaluate whether such mortality schedules are likely compared with other birds, or whether the low reproductive rates would require longer lifespans than is biologically plausible.

When demographic data are not available for the target species, PVA practitioners often substitute values from related taxa and acknowledge that the results must be treated with greater caution (e.g. Brook and Whitehead 2005). We initially used mortality data for Carnaby's Black-Cockatoo from Saunders (1982) as these are the most extensive mortality data for any species of black-cockatoo. We removed all human-induced deaths from this dataset (e.g. collision with vehicles) in order to establish the 'natural' background mortality rates. However, as discussed by Saunders, these reported rates of mortality are from wing-tagged birds that probably suffered higher than natural mortality. Saunders states that adult females in his study suffered higher mortality than males. We maintained this

Table 1. Demographic values used in the simulation models for Iron Range and Crater Mountain

\begin{tabular}{ll}
\hline Parameter & Value \\
\hline Initial population size & 1000 \\
$\begin{array}{l}\text { Carrying capacity } \\
\text { Age of first reproduction }\end{array}$ & 2000 \\
$\quad$ by males & 4 \\
Age of first reproduction & \\
$\quad$ by females & \\
Maximum age & 150 \\
$\begin{array}{l}\text { Mortality } \\
\text { Maximum progeny per year }\end{array}$ & See Table 2 \\
$\begin{array}{l}\text { Proportion males (sex-ratio) } \\
\quad \text { at hatching }\end{array}$ & 1 \\
$\begin{array}{l}\text { Percentage adult females breeding } \\
\text { No. of offspring per female per year }\end{array}$ & 0.5 \\
$\begin{array}{l}\text { Environmental variation (\%) } \\
\text { Percentage of males in breeding }\end{array}$ & Limited by hollow availability \\
$\quad$ population & $0.11 \pm 0.02$ (Iron Range) \\
Mating system & 10 \\
\hline
\end{tabular}


difference because female Palm Cockatoos invariably brood overnight when large pythons are active and are likely to be significant predators. We also maintained the relative difference between adults and juveniles in our models but progressively reduced all mortality rates until a model with stable nondecreasing population projections was attained. Four such modelling scenarios were examined to determine the possible mortality schedules (Table 2). The first model used the mortality rates of Saunders (1982) (corrected as above), the second model halved these values (i.e. multiplied by 0.5 ), the third halved them again $(0.25)$, and the fourth halved them again $(0.125)$. In the absence of accurate data we assume standard deviations of $\sim 20 \%$ of all values.

\section{Further limitations on breeding}

Tree-hollows for nesting are an important limiting resource for many parrots, including Palm Cockatoos (Snyder et al. 2004). In our models we allowed the percentage of females $(\mathrm{F})$ that breed each year to be based on the number of available hollows $(\mathrm{H})$ according to the VORTEX formula MIN $(1 ; \mathrm{H} / \mathrm{F}) \times 100$ where MIN takes the minimal value of the subsequent expression. This has the property of allowing all adult females to breed if there are sufficient hollows, or only as many females as there are available hollows. The 'MIN' function takes the smaller of these two values for each simulation.

The initial number of available hollows was set according to surveys undertaken by Murphy et al. (2003). There were 4.4 males $\mathrm{km}^{-2}$, and each male defended on average 3.4 tree-hollows. However, many of these (34 of 61 hollows) had different properties and were used exclusively for display purposes, such that the true number of potential breeding hollows was 1.5 per male. Therefore, the models for the initial population of 1000 Palm Cockatoos (500 males, 500 females) start with 750 hollows over the study area, representing 500 (i.e. 750/1.5) available breeding opportunities for females. Our models also allow for density dependent reproduction, and especially the low likelihood that males can defend multiple hollows as effectively when the hollows are at lower density. When the number of hollows drops below 500 over the study area, our

Table 2. Mortality rates (percentage mortality per year \pm s.d.) for males and females over five age-classes

Model 1 uses the corrected data from Saunders (1982)

\begin{tabular}{llccc}
\hline & Model 1 & Model 2 & Model 3 & Model 4 \\
& & $(0.5 \times$ Model 1) & $(0.25 \times$ Model 1 $)$ & $(0.125 \times$ Model 1) \\
\hline \multicolumn{5}{c}{ Female age (years) } \\
$0-1$ & $70.6 \pm 14.1$ & $35.2 \pm 7.0$ & $17.6 \pm 4.0$ & $8.8 \pm 2.0$ \\
$1-2$ & $37.1 \pm 7.4$ & $18.4 \pm 3.7$ & $9.2 \pm 2.0$ & $4.6 \pm 1.0$ \\
$2-3$ & $38.3 \pm 7.7$ & $19.6 \pm 3.9$ & $9.8 \pm 2.0$ & $4.9 \pm 1.0$ \\
$3-4$ & $36.4 \pm 7.0$ & $18 \pm 3.6$ & $9 \pm 2.0$ & $4.5 \pm 1.0$ \\
$4+$ & $36.4 \pm 7.0$ & $18 \pm 3.6$ & $9 \pm 2.0$ & $4.5 \pm 1.0$ \\
& \multicolumn{5}{c}{ Male age (years) } \\
$0-1$ & $70.6 \pm 14.1$ & $35.2 \pm 7.0$ & $17.6 \pm 4.0$ & $8.8 \pm 2.0$ \\
$1-2$ & $37.1 \pm 7.4$ & $18.4 \pm 3.7$ & $9.2 \pm 2.0$ & $4.6 \pm 2.0$ \\
$2-3$ & $38.3 \pm 7.7$ & $19.6 \pm 3.9$ & $9.8 \pm 2.0$ & $4.9 \pm 1.0$ \\
$3-4$ & $25.7 \pm 5.1$ & $13.2 \pm 2.6$ & $6.6 \pm 1.0$ & $3.3 \pm 1.0$ \\
$4+$ & $25.7 \pm 5.1$ & $13.2 \pm 2.6$ & $6.6 \pm 1.0$ & $3.3 \pm 1.0$ \\
\hline
\end{tabular}

models assume that males defend one hollow each in these circumstances. Thus each hollow becomes available for reproduction below this number.

Two further determinants of the number of available nesthollows were included in the models. First, owing to fire, wind and decay of wood, the number of hollows decreases by $\sim 5.2 \%$ per year. However, display hollows are often not as solid as true nesting hollows, and disappear faster, so that the true rate of loss of active nesting hollows is $\sim 1 \%$ per year (calculated from data in Murphy et al. 2003).

Offsetting this loss is the episodic creation of potential new hollows when high winds associated with cyclones break branches off trees, and open the inside of the tree to rot and termite activity (Murphy and Legge 2007). Although records for the exact frequency of cyclones hitting Iron Range are not available, meteorological records (Australian Bureau of Meteorology 2009) and discussions with local people (G. Pope, pers. comm.) suggest that cyclones severe enough to cause extensive damage to trees occur approximately every 20 years. From data supplied by Murphy and Legge (2007) we calculated that each cyclone creates a mean of 136 new hollows across the study area. This gradual loss and episodic addition of nesting hollows was programmed in VORTEX by first creating a 'catastrophe' that occurs every 20 years on average, and second, creating a global function that allowed both loss of hollows at $1 \%$ per year combined with the addition of new hollows when each cyclone hit the region. The number of hollows thus available each year was then used to determine the proportion of breeding females described above.

\section{Impact of inbreeding}

The role of inbreeding depression in the extinction of wild populations is controversial, because there are no quantitative estimates of its impact using realistic levels of inbreeding (O'Grady et al. 2006). To test if inbreeding had a possible detrimental impact; we ran models using three levels of the impact of inbreeding: none, moderate and high. Moderate inbreeding used the default value of 3.14 lethal recessives in VORTEX, which is the typical inbreeding level determined for mammals (Lacy 1993). High inbreeding used the value of 12 diploid lethals. This value was inferred from O'Grady et al. (2006) who determined that 12 diploid lethal equivalents of inbreeding would decrease the median times to extinction of birds by an average of $37 \%$ regardless of the carrying capacity. However, inbreeding for the Iron Range population of 1000 birds did not lead to significant decreases in population viability (see below), so no inbreeding was incorporated in the Crater Mountain models.

\section{Crater Mountain simulations}

Owing to lack of data, many Crater Mountain parameters were based on those for Iron Range. At the Crater Mountain study site, approximately one nest in six was observed to be active each year. This could suggest that pairs breed as infrequently as every six years, or that pairs defend multiple hollows and breed more frequently. However, as individuals were not marked, and we do not know how many nests each male guards, it is impossible to ascertain the true rate (Igag 2002). We consequently ran our 
models using both the higher Iron Range rate (every 2.2 years) and the lower rate of once per six years. At Crater Mountain, $40 \%$ of offspring were observed to fledge (Igag 2002), which is higher than the $22 \%$ for Iron Range. Mortality data were adapted from the likely scenario determined for Iron Range, but we include an additional source of mortality for nestlings in the Crater Mountain region due to the removal of chicks and eggs by people at a rate of $40 \%$ of nests each year (Igag 2002). No cyclones were imposed on this population as it is not in a cyclone-prone area. We assume that nests are created and destroyed at the same rate in the rainforest habitat, but owing to the lack of data do not include any further anthropogenic sources of loss of nests (e.g. clearing of habitat) in our models.

\section{Results}

\section{Iron Range}

Table 3 shows the simulated population trajectories for the Iron Range population using the four mortality scenarios based on data from Saunders' (1982). Model 1 (using original data from Saunders) shows a severely negative growth rate $(r=-0.33)$ with extinction in a median time of 16 years. Halving those mortality rates (Model 2) changed $r$ to -0.16 and increased the time to extinction to 39 years. Model $3(25 \%$ of mortality in scenario 1) changed $r$ to -0.05 . Although the population does not go extinct within 100 years under this scenario it is severely depleted to just 9.5 individuals $( \pm 4.5$ s.d.). A final reduction in mortality to 0.125 of Saunders' data (Model 4) yields a value of $r$ that is close to zero $(-0.001)$ and a final extant population of 900.5 individuals ( \pm 137.5 s.d.). The population trends of these four models are shown in Fig. 1. Because it yields a population roughly at equilibrium, we use Model 4 as our baseline model to explore the impact of other factors on population viability such as the effects of inbreeding and harvesting.

Figure 2 shows the age-structure of the Iron Range population under the conditions of Model 4. It shows a peak of birds aged 11-20 years with declining numbers in all older age-categories. To sustain their low reproductive rate, at least some Palm Cockatoos must be living past 90 years old. Two males in the simulation reached age 100 .

Halving the initial population size to 500 birds had little impact $(r=-0.002$ compared with -0.001$)$ on the population trajectory (Model 5). Similarly, neither moderate nor high inbreeding had major effects on either $r$ or the final population size using the parameters of Model 4 (Models 6 and 7, Table 3). This suggests that the starting population of 1000 birds is sufficiently large so that inbreeding does not have much effect. However both decreasing (Model 8) and increasing (Model 9) the age of first reproduction had major effects on population viability. In particular, an increase of the age of first breeding to five years reduced $r$ to -0.024 and increased the probability of extinction over 100 years from 0 to 0.08 (Table 3 ).

\section{Crater Mountain}

Assuming Palm Cockatoos at Crater Mountain breed at the same frequency as those at Iron Range, and with the same juvenile and adult mortality, the higher breeding success at Crater Mountain would lead to an increasing population size for Palm Cockatoos $(r=0.022)$ over 100 years (Model 1, Table 4). The increase in population growth rate in this scenario is limited by the fixed number of nest-hollows. The population size increases $(r=0.01)$ even if $20 \%$ of nests are harvested (Model 2). The population remains relatively stable $(r=-0.001)$ if $40 \%$ of nests are harvested (Model 3), and decreases markedly $(r=-0.014)$, with a probability of extinction of 0.018 , if $60 \%$ of nests are harvested (Model 4 ). If Palm Cockatoos breed only every six years on average, then the population shows a marked decrease $(r=-0.015)$, with a probability of extinction of 0.025 , even if there is no harvesting (Model 5). The population trends of these five models with population size at Years $0,25,50,75$, and 100 are shown in Fig. 3.

\section{Discussion}

Our analyses cast doubt on whether Palm Cockatoos have long enough lifespans to support the low rate of reproduction reported at Iron Range, and suggest instead that the population may be in decline. Here we discuss the strengths and weaknesses of our modelling approach, the likely lifespans of wild Palm Cockatoos, and whether the slightly higher rates of reproduction observed at the New Guinea field site allow for any level of sustainable harvesting of nestlings.

Table 3. The stochastic rate of population increase (r), probability of extinction $P(E)$, extant population size Pop(extant) (number of individuals $\pm \mathbf{s . d}$.), and the number of years to extinction under nine scenarios at Iron Range Model 5 uses the parameters of Model 4 to examine the effect of a smaller initial population (500 birds); Models 6 and 7 use the parameters of Model 4 to examine the effect of inbreeding severity; Models 8 and 9 use the parameters of Model 4 to examine the effect of age of first reproduction; n.a. = not applicable, as the number of years to extinction was only calculated when $P(\mathrm{E})=1$

\begin{tabular}{lcccc}
\hline Model & $r$ & $P(E)$ & Pop(extant) & Years to extinction \\
\hline Model 1 (no inbreeding) & -0.33 & 1.00 & $0 \pm 0$ & 16 \\
Model 2 (no inbreeding) & -0.16 & 1.00 & $0 \pm 0$ & 39 \\
Model 3 (no inbreeding) & -0.05 & 0.23 & $9.5 \pm 4.5$ & n.a. \\
Model 4 (no inbreeding) & -0.001 & 0 & $900.5 \pm 137.5$ & n.a. \\
Model 5 (initial population=500) & -0.002 & 0 & $402.2 \pm 59.0$ & n.a. \\
Model 6 (moderate inbreeding) & -0.001 & 0 & $900.9 \pm 134.1$ & n.a. \\
Model 7 (high inbreeding) & -0.001 & 0 & $885.1 \pm 138.7$ & n.a. \\
Model 8 (first breed at 3 years) & 0.031 & 0 & $1335.0 \pm 142.5$ & n.a. \\
Model 9 (first breed at 5 years) & -0.024 & 0.08 & $467.4 \pm 64.8$ & n.a. \\
\hline
\end{tabular}




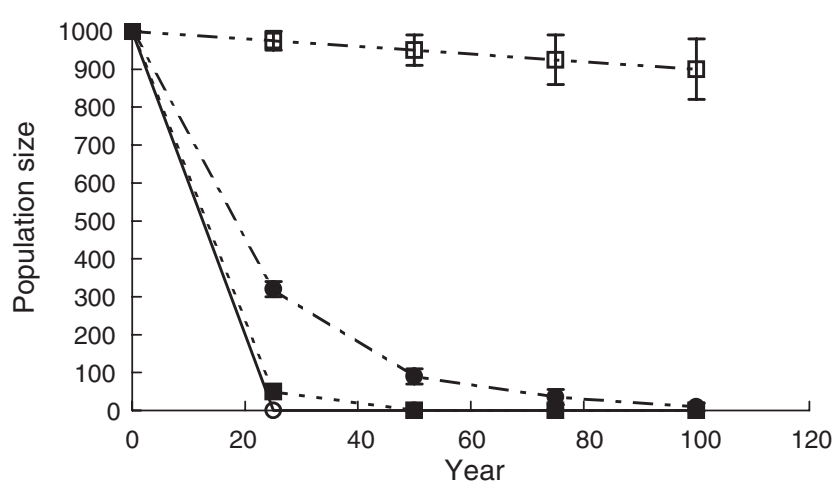

Fig. 1. Simulated population trajectories ( \pm s.d. $)$ at Iron Range, Queensland, under four scenarios of juvenile and adult mortality outlined in Table 2. Model $1=$ open circles, Model $2=$ closed squares, Model $3=$ closed circles, Model 4 = open squares.

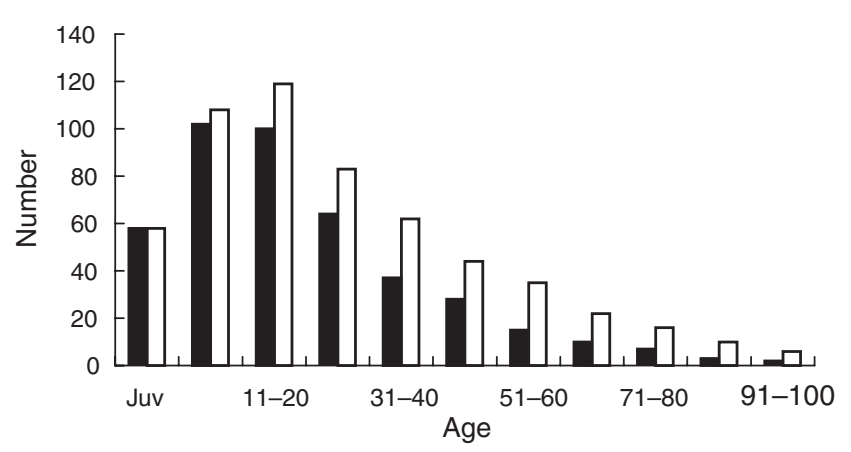

Fig. 2. Distribution of ages for females (black bars) and males (open bars) predicted for the Iron Range population using the mortality schedules of Model 4.

Table 4. The stochastic rate of population increase $(r)$, probability of extinction $P(E)$, and extant population size Pop(extant) (number of individuals \pm s.d.) using varying breeding intervals and rates of harvest at Crater Mountain

\begin{tabular}{lccccc}
\hline Model & $\begin{array}{c}\text { Breeding } \\
\text { interval }\end{array}$ & $\begin{array}{c}\text { Nest harvesting } \\
\text { rate }\end{array}$ & $r$ & $P(\mathrm{E})$ & Pop(extant) \\
\hline Crater 1 & 2 years & 0 & 0.022 & 0 & $1826.98 \pm 113.7$ \\
Crater 2 & 2 years & $20 \%$ & 0.010 & 0 & $1737.51 \pm 137.7$ \\
Crater 3 & 2 years & $40 \%$ & -0.001 & 0 & $923.91 \pm 179.3$ \\
Crater 4 & 2 years & $60 \%$ & -0.014 & 0.018 & $54.96 \pm 54.9$ \\
Crater 5 & 6 years & 0 & -0.015 & 0.025 & $231.64 \pm 55.7$ \\
\hline
\end{tabular}

\section{Model assumptions}

The PVA approach provides a valuable tool for determining the likely trajectory and long-term viability of populations, but as for any modelling exercise the assumptions and quality of data must be explicitly evaluated (Coulson et al. 2001). The strength of our modelling approach is that it relies on robust field data on both reproductive success and changing availability of nest-hollows over multiple years. This allows the interaction between these two major limitations on breeding and population growth to be captured explicitly. Nesting hollows appear to be a hotly contested resource at Iron Range and Crater Mountain with

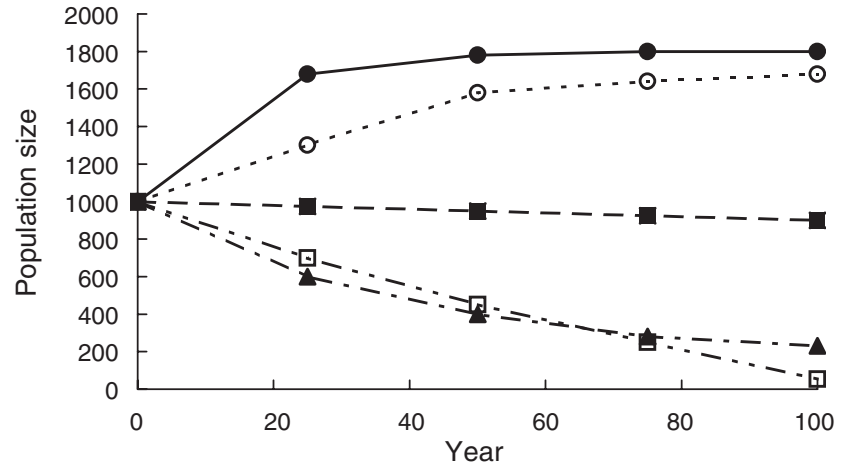

Fig. 3. Simulated population trajectories ( \pm s.d.) at Crater Mountain, PNG, under the five scenarios of breeding frequency and nest harvesting rates outlined in Table 4 . Crater $1=$ closed circles, Crater $2=$ open circles, Crater $3=$ closed squares, Crater $4=$ open squares, Crater $5=$ closed triangles.

many fights between Palm Cockatoos (Igag 2002; Murphy et al. 2003), and also between them and other species (Heinsohn et al. 2003). Our model is flexible in that it limits the number of breeding females to the total number of breeding opportunities. These may be limited by males when they defend multiple hollows, or by the actual number of hollows when they are scarce. The number of hollows fluctuates with losses from fire, wind and rot, and episodic replacement through cyclones (Murphy and Legge 2007), leading to a variable resource base that interacts with other aspects of Palm Cockatoo reproduction. One noteworthy implication of this variability is that the incidence of reproductive failure resulting from intraspecific and interspecific interference competition over limited nesthollows may also vary. We observed direct and circumstantial evidence of interference competition during the course of fieldwork at both study sites, although it was difficult to determine precisely how often it caused breeding failure (Murphy et al. 2003). Nevertheless, such variation would mean that during times when hollows are abundant (for example, following a tropical cyclone) interference competition may relax with a concomitant increase in reproductive success. However, we do not believe that this relaxation could ever compensate for the high rate of predation of eggs and nestlings and therefore overall reproductive success, even during peak availability of hollows, is likely to remain low.

Our models only deal with the hollows that are actually used for breeding. Each male has on average 1.5 such hollows on his territory, but also uses a variety of other hollows for display purposes. Whereas nest-hollows are destroyed at $\sim 1 \%$ per year, display hollows disappear at a higher rate (Murphy and Legge 2007). We do not know whether a male is inhibited from breeding without these specific display hollows, and as these turn over at a higher rate this may constitute an additional dynamic not captured in our model.

Knowledge of these key limitations to breeding allow exploration of other demographic variables for which there is less information, particularly age at first reproduction, and juvenile and adult mortality. An important assumption in our models is that wild Palm Cockatoos breed by the time they are four years old. This appears to be supported by data from species of black-cockatoo (Calyptoryhnchus) (Saunders 1982, 1986). 
One species, the Glossy Black-Cockatoo (Calyptoryhnchus lathami), can breed even earlier (Garnett et al. 1999). Our sensitivity analysis of this variable suggested that it is of critical importance. Even shifting the breeding age of Palm Cockatoos by one year to age five leads to a marked decrease in the stochastic growth rate $(r)$ from $\sim 0$ to -0.024 (Model 9 , Table 3), confirming that this life-history parameter needs further study in the field. Our models are also hampered by poor estimates of the variation around certain parameters. For example, although the estimate of reproductive rate is uncommonly good for such a difficult-to-study and long-lived species, and allows excellent deterministic projections, a longer field-based sample would allow greater appreciation of stochastic fluctuations.

In the absence of appropriate data for Palm Cockatoos, the models made use of a dataset for mortality collected over seven years by Saunders (1982) for individually marked Carnaby's Black-Cockatoos in Western Australia. Although they live in a different habitat type these birds show similar life-history traits to Palm Cockatoos, including small clutch-size (two eggs, but usually only one surviving) and low rates of breeding success. However, as discussed by Saunders (1982) the rates of mortality for both adults and juveniles appeared to be overestimated in that study, probably owing to the heightened conspicuousness of birds with wing-tags. We nonetheless use these data in our models both to establish the relative rates of mortality between adults and juveniles, and as a starting point for determining how low mortality rates must be for the Palm Cockatoo populations to be stable. As noted above, we are confident in the higher female mortality rate that we invoked in the models because they brood overnight when large predators are most active.

Although there is a tendency for middle-aged birds to reproduce and survive better than either young or old birds (Newton 1989) our models assume that Palm Cockatoos do not show either increased mortality or reproductive senescence even at very old ages. Although data are scarce for parrots, some seabirds exhibit exceptionally slow age-related declines in survival, and some also show negligible reproductive senescence. Reproductive success in the California Gull (Larus californicus) even increases in older pairs until they disappear from the breeding population (Pugesek and Diem 1990). Such slow ageing rates are limited to species with life histories characterised by delayed sexual maturation and very small clutch-sizes, such as those found in the Cacatuidae.

\section{Do Palm Cockatoos live long enough?}

On available evidence it seems unlikely that Palm Cockatoos at Iron Range live long enough on average to support their low rate of reproduction. In other words, our field data showing extremely low reproductive success (Murphy et al. 2003) may also be indicative of population decline. Our models suggest that population stability over 100 years would rely on very low mortality rates of juveniles (e.g. 8.8\%) and adults (e.g. 3.3-4.9\%), which are much lower than the rates reported for Carnaby's Black-Cockatoos (25.7-70.6\%). Palm Cockatoos, like other members of the Cacatuidae (Munshi-South and Wilkinson 2006), can be very long lived, and the oldest recorded Palm
Cockatoo in captivity was 56 years old (Brouwer et al. 2000). Although our models for wild Palm Cockatoos at Iron Range suggest that most of the population would indeed be less than 60 years old, there is nonetheless a long tail on the distribution with a small but important number of birds living up to 100 years old (Fig. 2). It is also important to remember that this comparison is based only on the single longest-lived Palm Cockatoo rather than mean mortality schedules from a larger sample of captive birds, and that captive animals presumably live longer than wild animals because they do not face natural predators (Brouwer et al. 2000). The discrepancy between our model and the known maximum age may also be a result of uncertainty over various demographic traits (see above), and errors in our field data (e.g. owing to small sample sizes and the possibility of missed reproductive events). We nonetheless stress that available evidence suggests that reproductive success does not balance mortality at Iron Range, and that the population there may be in decline. As the Iron and McIlwraith Ranges represent the largest block of rainforest habitat on Cape York Peninsula, this region is also unlikely to be a 'sink' for other populations.

Previous discussions of the conservation status of Palm Cockatoos have noted their slow life history and the consequent likely sensitivity to environmental perturbations such as the loss of nest-hollows and breeding habitat through inappropriate fire regimes (Frith and Frith 1993; Murphy et al. 2003). However, this study suggests that their low reproductive success might in itself be a cause for concern. The three largest causes of nesting failure were predation of nestlings $(37.9 \%)$, predation of eggs $(24.1 \%)$ and infertility of eggs (17.2\%) but we are currently unable to evaluate if any of these factors are artificially elevated. Predation of eggs and both small and large nestlings was apparently carried out by a suite of natural predators including large rodents, butcherbirds, small and large varanids, and pythons. Alternatively, egg infertility could suggest deleterious effects of inbreeding (O'Grady et al. 2006). Whereas even the most severe of our inbreeding models suggested that inbreeding does not have a major impact on the Iron Range population, these analyses were based on an assumed population size of 1000 birds. In the absence of any clear indication of anthropogenic factors affecting predator numbers and breeding success, we urge further monitoring of reproductive success and individual mortality rates in the population of Cape York Peninsula. We also stress the need for better data on population size in case the populations are small enough to suffer the effects of inbreeding.

\section{Are current rates of nest-harvesting at Crater Mountain sustainable?}

Many rural people in New Guinea rely on hunted animals for food. A recent analysis suggested that in PNG as much as 10000 tonnes of meat from wild animals may be hunted each year (Mack and West 2005). Most of the biomass comes from larger species such as wild Pigs (Sus scrofa) and Southern Cassowaries (Casuarius casuarius) but many smaller species of vertebrates are also taken. Mack and West (2005) stress that many species may be in serious decline through growing human populations and economic pressures (see, for example, Johnson et al. 2004) and have stressed the need for further research on 
the more vulnerable species, such as those with low reproductive rates. Palm Cockatoos are hunted opportunistically throughout New Guinea, and at Crater Mountain most of the individuals taken for food are nestlings. Igag (2002) determined that $40 \%$ of nestlings are taken by local people in the CMWMA. Often hunters secure cavity-using animals by chopping down the entire tree, thereby further limiting the number of suitable cavities to the overall population of cavity-dwelling species (A. Mack, pers. obs.).

Our analysis casts doubt on the sustainability of these current rates of harvest but does not rule out that some lower rate might be sustainable. The central model using the mortality schedules necessary for population stability at Iron Range suggests that the population could be stable over 100 years if $40 \%$ of nestlings are removed, and that the population might even increase if the harvest is decreased to $20 \%$ of nestlings. However, this is probably unrealistic for several reasons. First, it is unlikely that Palm Cockatoos live as long as the Iron Range models suggest (see above). Second, owing to a larger variety of predators, especially raptors (e.g. New Guinea Eagle (Harpyopsis novaeguineae); Igag 2002), predation rates of adult Palm Cockatoos may be higher in New Guinea than on Cape York Peninsula. Such additional extrinsic mortality could balance any favourable effect of the higher breeding success observed by Igag (2002). Third, we do not know the rate at which females attempt to breed at Crater Mountain. Again any advantage of higher breeding success will be quickly eroded if the rate is lower than at Iron Range (Igag 2002).

It is interesting that mortality schedules and breeding rates of Palm Cockatoos appear to differ between Cape York Peninsula and New Guinea, and that our preliminary models suggest that some level of hunting might be sustainable even in such a slowly reproducing species. The New Guinea populations have after all been hunted at some level for many thousands of years. We echo Mack and West's (2005) call for more research across New Guinea on sustainable rates of animal harvests, especially in light of the growing human population and the likely increased economic reliance on wildlife. Until better data are available we strongly urge overall harvesting of Palm Cockatoo nestlings be cut drastically. As it is not a target species and rates of harvest on such a low density species are difficult to impose and monitor, the best interim solution might be to ask local people to avoid harvesting them altogether.

In summary, our use of currently available demographic data in population viability analyses suggests that Palm Cockatoos at Iron Range on Cape York Peninsula are unlikely to live long enough on average to support their low rates of reproduction. We thus highlight the possibility that the population may be in decline and urge further research and monitoring of population trends both at Iron Range and in other parts of their range on Cape York Peninsula. At Crater Mountain, where nestlings are harvested for food by local people, the models suggest that the reported current rate of $40 \%$ of nestlings taken is not sustainable. We also note that our models do not explicitly account for loss of habitat (e.g. logging in New Guinea, changed fire regimes on Cape York Peninsula), other losses such as illegal trapping for the pet trade, or the potential effects of climate change, and therefore may overestimate population viability in some cases. We consequently suggest that, until more detailed and longer term data are available, Palm Cockatoos be moved from the category of 'Least Concern' of the Queensland Environmental Protection Agency to 'Vulnerable', and from 'Least Concern' on the International Union for Conservation of Nature Red List (IUCN 2008) to 'Near Threatened'.

\section{Acknowledgements}

We thank Naomi Langmore and three anonymous reviewers for helpful comments on the manuscript, and Bob Lacy and Phil Miller for technical assistance with VORTEX. Our research on Palm Cockatoos has been supported by grants from the World Parrot Trust, the Hermon Slade Foundation, and through excellent logistical support from the Fenner School of Environment and Society, Australian National University.

\section{References}

Austad, S. N. (1993). The comparative perspective and choice of animal models in aging research. Aging Clinical and Experimental Research 5, 259-267.

Austad, S. N., and Fischer, K. E. (1991). Mammalian aging, metabolism, and ecology: evidence from the bats and marsupials. Journal of Gerontology 46, B47-B53.

Australian Bureau of Meteorology (2009). Tropical cyclones in Queensland: Historical impacts along the east coast. Available at http://www.bom. gov.au/weather/cyclone/about/cyclones-eastern-impacts.shtml [Verified 1 July 2009].

Beissinger, S. R., and Westphal, M. I. (1998). On the use of demographic models of population viability in endangered species management. Journal of Wildlife Management 62, 821-841. doi: 10.2307/3802534

BirdLife International (2009). What's new (2009). Available at http://www. birdlife.org/action/science/species/global_species_programme/whats_ new.html [Verified 1 July 2009].

Brook, B. W., and Whitehead, P. J. (2005). Plausible bounds for maximum rate of increase in magpie geese (Anseranas semipalmata): implications for harvest. Wildlife Research 32, 465-471. doi: 10.1071/WR02105

Brouwer, K., Jones, M. L., King, C. E., and Schifter, H. (2000). Longevity records for Psittaciformes in captivity. International Zoo Yearbook 37, 299-316. doi: 10.1111/j.1748-1090.2000.tb00735.x

Cahill, A. J., Walker, J. S., and Marsden, S. J. (2006). Recovery within a population of the critically endangered citron-crested cockatoo Cacatua sulphurea citrinocristata in Indonesia after 10 years of international trade control. Oryx 40, 161-167. doi: 10.1017/S0030605306000366

Coulson, T., Mace, G. M., Hudson, E., and Possingham, H. (2001). The use and abuse of population viability analysis. Trends in Ecology \& Evolution 16, 219-221. doi: 10.1016/S0169-5347(01)02137-1

Frith, C. B., and Frith, D. W. (1993). Notes on birds found nesting at Iron Range, Cape York Peninsula, November-December 1990. Sunbird 23, 44-57.

Garnett, S., Pedler, L. P., and Crowley, G. M. (1999). The breeding biology of the Glossy Black-Cockatoo Calyptoryhnchus lathami on Kangaroo Island, South Australia. Emu 99, 262-279. doi: 10.1071/MU99032

Heinsohn, R., Murphy, S., and Legge, S. (2003). Overlap and competition for nest holes among eclectus parrots, palm cockatoos and sulphur-crested cockatoos. Australian Journal of Zoology 51, 81-94. doi: 10.1071/ ZO02003

Holmes, D. J., Fluckiger, R., and Austad, S. N. (2001). Comparative biology of aging in birds: an update. Experimental Gerontology 36, 869-883. doi: 10.1016/S0531-5565(00)00247-3

Igag, P. (2002). The conservation of large rainforest parrots: a study of the breeding biology of palm cockatoos, eclectus parrots and Pesquet's parrots. M.Sc. Thesis, Australian National University, Canberra.

IUCN (2008). '2008 IUCN Red List of Threatened Species.' (International Union for Conservation of Nature.) Available at www.iucnredlist.org [Verified 1 July 2009]. 
Johnson, A., Bino, R., and Igag, P. (2004). A preliminary evaluation of the sustainability of cassowary (Aves: Casuariidae) capture and trade in Papua New Guinea. Animal Conservation 7, 129-137. doi: 10.1017/ S1367943003001173

Juniper, T., and Parr, M. (1998). 'Parrots: A Guide to Parrots of the World.' (Pica Press: Sussex, UK.)

Lacy, R. C. (1993). VORTEX: A computer simulation model for Population Viability Analysis. Wildlife Research 20, 45-65. doi: 10.1071/WR993 0045

Mack, A. L., and West, P. (2005). Ten thousand tonnes of small animals: Wildlife consumption in Papua New Guinea, a vital resource in need of management. Resource Management in Asia-Pacific Working Paper No. 61. Australian National University, Canberra.

Miller, P. S., and Lacy, R. C. (2005). 'Vortex: A Stochastic Simulation of the Extinction Process. Version 9.50 User's Manual.' (Conservation Breeding Specialist Group (Species Survival Commission and International Union for Conservation of Nature): Apple Valley, MN.)

Munshi-South, J., and Wilkinson, G. S. (2006). Diet influences life span in parrots. Auk 123, 108-118. doi: 10.1642/0004-8038(2006)123[0108: DILSIP]2.0.CO;2

Murphy, S., and Legge, S. (2007). The gradual loss and episodic creation of Palm Cockatoo (Probosciger aterrimus) nest-trees in a fire- and cycloneprone habitat. Emu 107, 1-6. doi: 10.1071/MU06012

Murphy, S., Legge, S., and Heinsohn, R. (2003). The breeding biology of palm cockatoos (Probosciger aterrimus): a case of a slow life history. Journal of Zoology 261, 327-339. doi: 10.1017/S0952836903004175

Nelder, V., and Clarkson, J. (1995). 'Vegetation Survey of Cape York Peninsula. Cape York Peninsula Land Use Strategy.' (Department of Environment and Heritage: Brisbane.)

Newton, I. (1989). 'Lifetime Reproduction in Birds.' (Academic Press: London, UK.)
O'Grady, J. J., Brook, B. W., Reed, D. H., Ballou, J. D., Tonkyn, D. W., and Frankham, B. (2006). Realistic levels of inbreeding depression strongly affect extinction risk in wild populations. Biological Conservation 133, 42-51. doi: 10.1016/j.biocon.2006.05.016

Prinzinger, R. (1993). Life span in birds and the ageing theory of absolute metabolic scope. Comparative Biochemistry and Physiology 105A, 609-615.

Pugesek, B. H., and Diem, K. L. (1990). The relationship between reproduction and survival in known-aged California gulls. Ecology 71, 811-817. doi: $10.2307 / 1940332$

Saunders, D. A. (1982). The breeding behaviour and biology of the shortbilled form of the white-tailed black cockatoo Calyptorhynchus funereus. Ibis 124, 422-455. doi: 10.1111/j.1474-919X.1982.tb03790.x

Saunders, D. A. (1986). Breeding season, nesting success, and nestling growth in Carnaby's Cockatoo, Calyptorhynchus funereus latirostris, over 16 years at Coomallo Creek, and a method for assessing the viability of populations in other areas. Australian Wildlife Research 13, 261-273. doi: 10.1071/WR9860261

Snyder, N., McGowan, P., Gilardi, J., and Grajal, A. (2004). 'Parrots: Status Survey and Conservation Action Plan 2000-2004.' (International Union for Conservation of Nature: Gland, Switzerland.)

Walker, J. S., Cahill, A. J., and Marsden, S. J. (2005). Factors influencing nestsite occupancy and low reproductive output in the Critically Endangered Yellow-crested Cockatoo Cacatua sulphurea on Sumba, Indonesia. Bird Conservation International 15, 347-359. doi: 10.1017/S09592709 05000638

Manuscript received 4 October 2008, accepted 16 April 2009 